\title{
Finite element design procedure for correcting the coining die profiles
}

\author{
Paulo Alexandrino ${ }^{1}$, Paulo J Leitão ${ }^{1}$, Luis M Alves ${ }^{2}$, and Paulo A.F. Martins, ${ }^{2}{ }^{*}$ \\ ${ }^{1}$ INCM, Imprensa Nacional Casa da Moeda, Av. António José de Almeida, 1000-001 Lisboa, Portugal \\ 2 IDMEC, Instituto Superior Técnico, Universidade de Lisboa, Av. Rovisco Pais, 1049-001 Lisboa, Portugal
}

Received: 15 February 2018 / Accepted: 16 March 2018

\begin{abstract}
This paper presents a new finite element based design procedure for correcting the coining die profiles in order to optimize the distribution of pressure and the alignment of the resultant vertical force at the end of the die stroke. The procedure avoids time consuming and costly try-outs, does not interfere with the creative process of the sculptors and extends the service life of the coining dies by significantly decreasing the applied pressure and bending moments. The numerical simulations were carried out in a computer program based on the finite element flow formulation that is currently being developed by the authors in collaboration with the Portuguese Mint. A new experimental procedure based on the stack compression test is also proposed for determining the stress-strain curve of the materials directly from the coin blanks.
\end{abstract}

Keywords: Coining / dies / finite element modelling / experimentation

\section{Introduction}

Coin minting is a net-shape metal forming process in which a disk (coin blank) is compressed between dies whilst it is being retained and positioned by a collar (Fig. 1). The research in science and technology of coin minting can be divided into three main periods that extended over the past five decades. The first period (1960-1980) draws on the pioneering investigations of Bocharov et al. [1] and Bay and Wanheim [2] to the development of a theoretical framework for understanding the mechanics of coin minting by Kiran and Shaw [3]. The first two investigations were focused on the required stress to form a single isolated groove [1] and on the material displaced by adjacent features (asperities) [2], and gave rise to the characterization of coin minting as a three stage forming process involving indentation, gross upsetting and interaction between adjacent relief coin features [3].

The work of Kiran and Shaw [3] considered the coin surfaces to be approximated by simple saw tooth relief profiles characterized by a pitch and a feature included angle under plane strain deformation conditions. In fact, most of the investigations performed in this period were limited to simple triangular and semi-circular relief coin features and were exclusively based on analytical models built upon the slab, slip-line and upper-bound methods.

\footnotetext{
* e-mail: pmartins@tecnico.ulisboa.pt
}

The second period (1980-2000) enhanced previous knowledge on the deformation mechanics of coin minting by giving special attention to the analysis of material flow and calculation of pressure. Delamare and Montmitonnet [4] made use of the upper bound method to characterize plastic deformation inside a coin and to calculate the energy and the shape of a disk to produce a coin with a central circular design and an outer annular legend. Brekelmans et al. [5] compared the predictions of material flow and coining pressure for producing a conical relieve feature in the centre of a coin obtained. The work made use of the upper bound method and of the finite element method built upon a quasi-static formulation with elastoplastic constitutive equations, and is considered the first application of the finite element method in coin minting.

Subsequent applications of the finite element method under axisymmetric material flow conditions by Barata Marques and Martins [6] and by Leitão et al. [7] focused on the joining of bimetallic ring coins and on the development of a multi-stage coin minting process to produce bimetallic foil coins. The multi-stage coin minting technology was protected by an international patent [8].

The first three-dimensional application of the finite element method in coin minting was reported by Choi et al. [9] who developed a computer program based on a quasistatic formulation and rigid-plastic constitutive equations. The program was applied to the piercing and coining of a hole at the centre of a disk but no comparison was given between the numerical estimates and the actual material flow. The second period (1980-2000) ended with the 


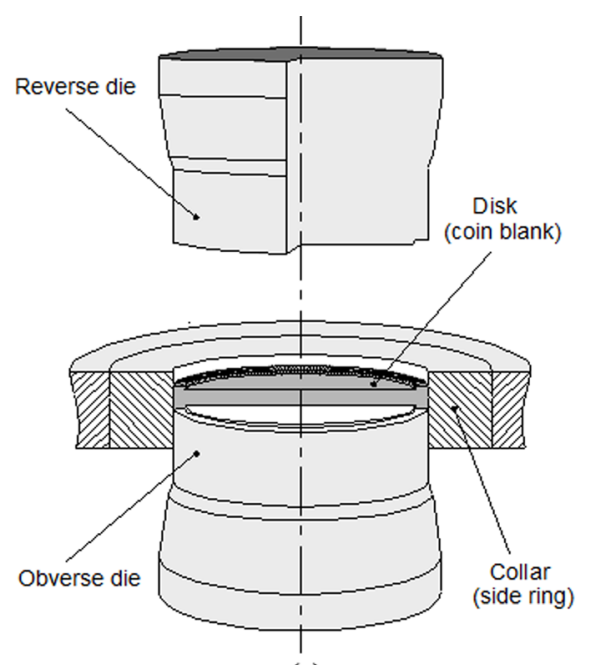

(a)

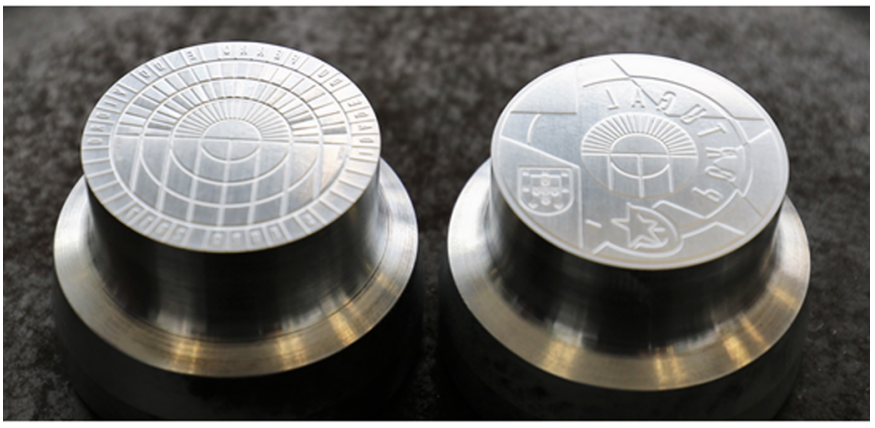

(b)

Fig. 1. Coin minting. (a) Schematic representation and notation, (b) Photograph of the reverse and obverse dies that were utilized to produce the collection coin dedicated to the age of iron and glass in Europe ("coin A").

investigation of Ike and Plancak [10] on the material flow to imprint coin microfeatures, which included a comparison between the experimentally required contact pressure and that calculated by means of the upper bound method.

The third period (2000-until now) has been mainly driven by the availability of three-dimensional finite element computer programs and high performance computers that allow accounting for the complex contact features that are typical of coin minting in order to produce accurate predictions of material flow, distribution of strain, stress and damage and evolution of the force with die stroke.

In 2007, Buffa et al. [11] utilized a commercial threedimensional finite element computer program based on a quasi-static formulation with rigid-plastic constitutive equations to simulate coin minting. The disk was discretized by means of a non-structured mesh of tetrahedral elements and the dies and collar were assumed to be rigid objects. Only simple three-dimensional circular, o-shaped and t-shaped relief features were engraved in the coin. The accuracy and reliability of the numerical predictions were not validated against experimental results.

In 2008, Xu et al. [12] reported the development of a special-purpose finite element computer program based on a dynamic explicit formulation with elasto-plastic consti- tutive equations to simulate coin minting. The discretization of the disks was performed by means of hexahedral elements and the dies and collar were considered rigid and discretized by means of spatial triangular and quadrilateral elements. The program was utilised to optimize the edge shape of the disks.

In 2009, Guo [13] utilized a commercial threedimensional finite element computer program based on a dynamic explicit formulation to simulate coin minting with serrations on the inner surface of the collar. The dies were considered rigid and discretized by means of triangular elements, but the overall level of detail and complexity of its surfaces was simplified by removing letters and replacing the relief features of the figures by stepped cavities with $0.1 \mathrm{~mm}$ depth. The numerical predictions of the coin minting force obtained under these simplifying assumptions were compared against experimental results but there were no conditions to compare the finite element predicted and experimentally observed filling of the actual relief coin features.

Later in 2012, Zhong et al. [14] utilized the above mentioned special-purpose finite element computer program [12] to predict the formation of tiny scratches caused by radial friction in the flat regions of the coin adjacent to the edge. The accuracy and reliability of the computer program was assessed by means of qualitative observations related to material flow. Quantitative comparisons between finite element predictions and experimental results were limited.

Then, Li et al. [15] further developed the special purpose dynamic explicit finite element computer program [12] by introducing locking-free hexagonal elements and an adaptive refinement strategy aimed at increasing the number of elements near the fine relief coin features. The overall strategy was applied in the numerical simulation of a simple coin and of a 'key-shaped' commemorative coin made from $99.9 \% \mathrm{Ag}$. Experimental validation of the force with displacement was provided for the 'key-shaped' commemorative coin.

Also in 2016, Shirasaka [16] revealed that Japan Mint is currently using the finite element method to modify relief coin features in order to increase die life. The work reports an increase of die life up to 2.5 times when the relief height is reduced to $60 \%$.

This paper presents a new finite element based design procedure for correcting the coining die profiles in order to optimize the distribution of pressure and the alignment of the resultant vertical force at the end of the die stroke. The paper is organized in five sections, including this introduction (Section 1). Section 2 presents a brief overview of the finite element computer program that is being developed by the authors in collaboration with the Portuguese Mint [17] and describes the methodology for transforming the three-dimensional models prepared by the sculptor into the triangular spatial meshes that are utilized in the discretization of the dies. Section 3 is focused on experimentation and presents a new procedure based on the stack compression test to determine the stress-strain curve of the materials utilized in coin minting directly from the coin blanks. This section also describes a simple experimental procedure for validating the force values 


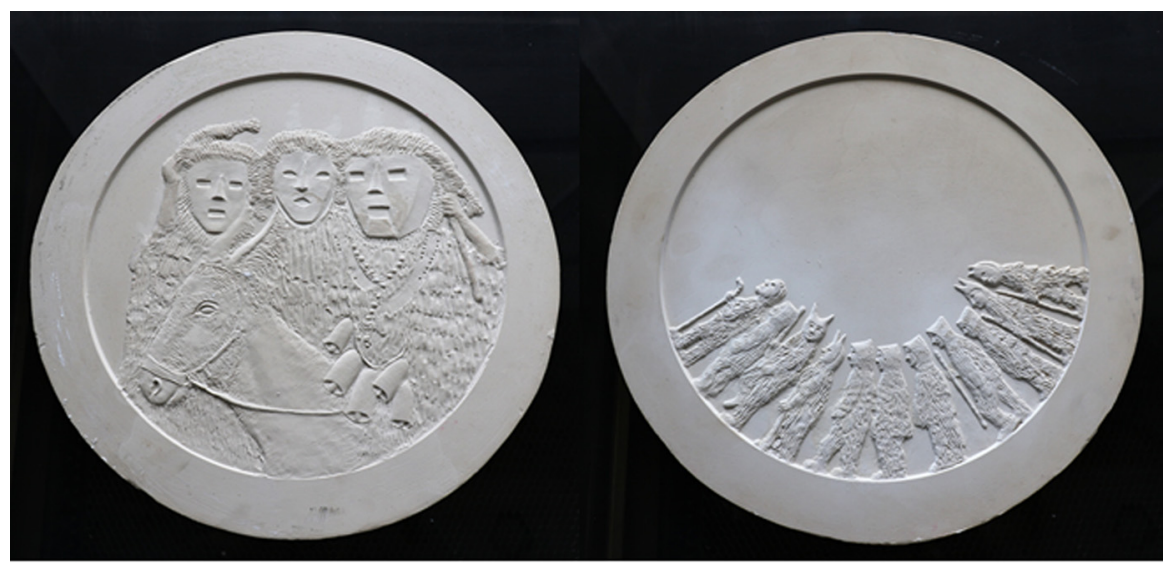

(a)
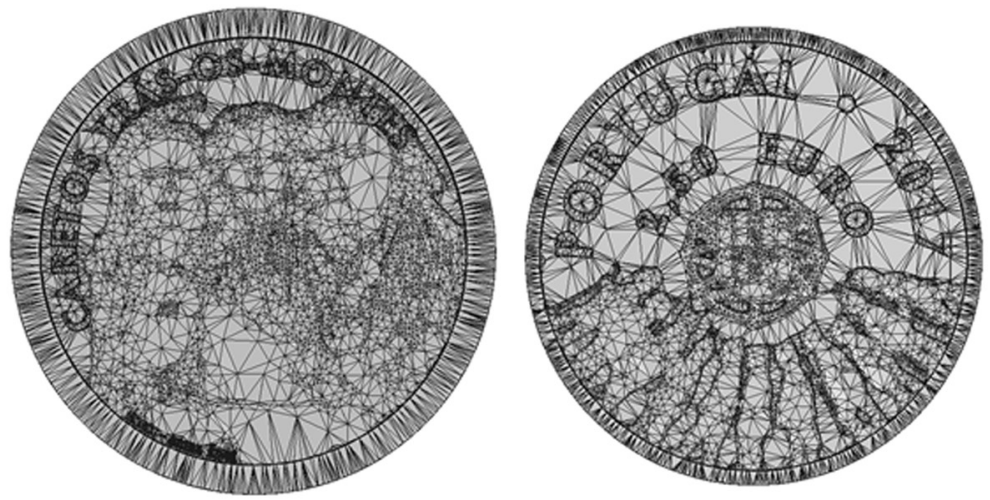

(b)

Fig. 2. Discretization of the die surfaces. (a) Original clay models supplied by the sculptor (scale 7:1), (b) Discretization of the die surfaces by means of spatial triangular elements after scanning and scaling down the clay models to the actual coin size.

given by the industrial coin minting press. Section 4 presents and discusses the results. The first part deals with the accuracy and reliability of the results provided by the finite element computer program. Numerical predictions of the progressive filling of the relief coin features and of the force vs. die stroke evolution are compared with experimental measurements and observations. The second part introduces the finite element based design procedure for correcting the coining die profiles and demonstrates its effectiveness to optimize the distribution of pressure and the alignment of the resultant vertical force at the end of the die stroke. Finally, Section 5 presents the conclusions.

\section{Finite element modelling}

The finite element computer program that is being developed by the authors in collaboration with the Portuguese Mint is built upon the three dimensional finite element computer system I-form, which is based on the irreducible finite element formulation and accounts for the contact with friction between rigid and deformable objects [18],

$$
\begin{aligned}
\Pi= & \int_{V} \bar{\sigma} \dot{\bar{\varepsilon}} d V+\frac{1}{2} K \int_{V} \dot{\varepsilon}_{v}^{2} d V-\int_{S_{T}} t_{i} u_{i} d S \\
& +\int_{S_{f}}\left(\int_{0}^{\left|u_{r}\right|} \tau_{f} d u_{r}\right) d S
\end{aligned}
$$

In the above functional, $\bar{\sigma}$ is the effective stress, $\dot{\bar{\varepsilon}}$ is the effective strain rate, $\dot{\varepsilon}_{V}$ is the volumetric strain rate, $K$ is a large positive constant imposing the incompressibility of volume $V, t_{i}$ and $u_{i}$ are the surface tractions and velocities on surface $S_{T}, \tau_{f}$ and $u_{r}$ are the friction shear stress and the relative velocity on the contact interface $S_{f}$ between the coin blanks and the dies or collar. Friction is modelled through the utilization of the law of constant friction $\tau_{f}=m k$. A detailed description of the computer implementation of the finite element flow formulation is given in Nielsen et al. [19].

Figure 2 shows the original clay models (in a scale $7: 1$ ) prepared by the sculptor and the discretization of the corresponding reverse and obverse dies by means of spatial triangular contact-friction elements. The collar with a serrated profile (not shown in Fig. 2) was also discretized by means of spatial triangular contact-friction elements.

The original clay models are usually much larger than the dies in order to allow facial details, hair and hands to be sculptured more lifelike and also to facilitate the inclusion of folds and other realistic features in cloths, flowers and other motifs commonly available in coins. In fact, all these details would be very difficult to accomplish if the sculptor theme had to be directly expressed in the actual size of the coins.

The scaling down of the clay models to the actual shape and size of the dies is performed digitally and involves 


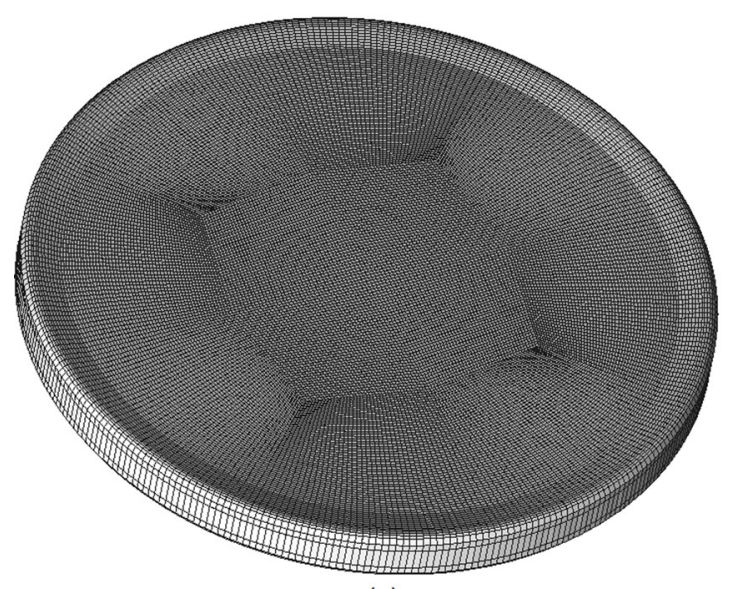

(a)

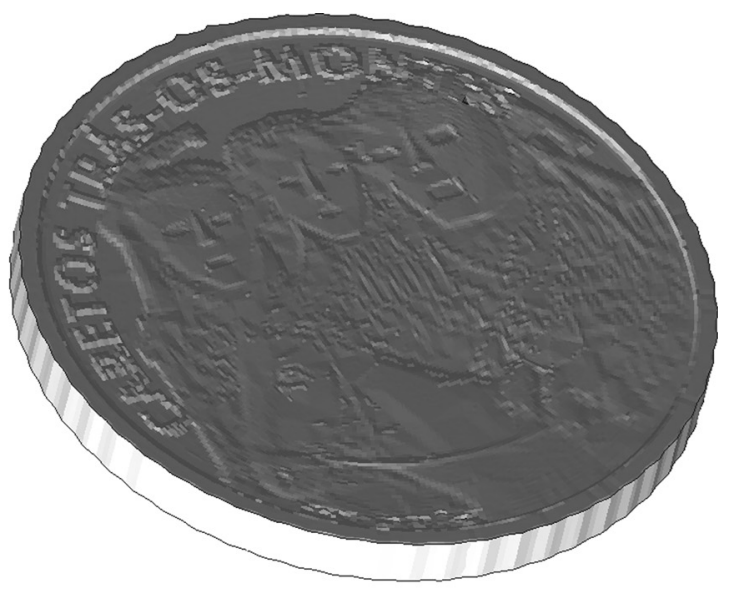

(b)

Fig. 3. Finite element simulation of coin minting. (a) Discretization of the coin blank by hexahedral elements, (b) Numerically predicted geometry of the coin at the end of the process.

three-dimensional scanning and correction of the relief profiles in order to add appropriate small convex curvatures on the reverse and obverse surfaces of the dies. Letters, numbers and the national coat of arms are digitally added to each die.

The digital representation of each die surface is performed by means of a triangular tessellation and then saved in an STL (stereolithography) file format and converted into a workable finite element input data file. The conversion of the STL file into a workable finite element input data file containing the geometry of the dies is performed automatically by the finite element computer program in order to save time and effort and to prevent the loss of details of the die profiles due to the utilization of intermediate pre-processing systems.

The coin blanks are discretized by a structured mesh of hexahedral elements with 5 layers of elements across thickness (Fig. 3). The choice of hexahedral elements instead of tetrahedral elements is due to fact that tetrahedral elements are overly stiff, very much sensitive to mesh orientation and also because the finite element simulation of coin minting does not require remeshing.

In addition, it is worth noticing that published research work in the field of structural mechanics usually points out to the necessity of tetrahedral elements frequently requiring up to an order of magnitude more elements to achieve the same level of accuracy as alternative hexahedral elements [20,21]. In other words, meshing of coin blanks by means of hexahedral elements is likely to decrease the overall element count and, therefore, to ensure faster CPU times. This justifies the choice of hexahedral elements to discretize the coin blanks.

The finite element simulations of coin minting were performed with a convergence tolerance $\eta$ of the residual force vector equal to $10^{-3}$ and the overall numerical simulation is accomplished through a succession of approximately 100 displacement increments each of one modelling approximately $0.1 \%$ of the initial thickness of the coin blanks.
Typical CPU times for the computer simulation of coin blanks with 100.000 elements is between $12 \mathrm{~h}$ and $18 \mathrm{~h}$, depending on the complexity of the die surfaces. Coin blanks with 160.000 elements usually require 3 times more CPU time.

\section{Experimentation}

The case studies included in this paper are the collection coins dedicated to the age of iron and glass in Europe (Fig. 4a, hereafter designated as "coin A") and to Portuguese Ethnography (Fig. 4b, hereafter designated as "coin B"). Table 1 summarizes the material and geometry of the disks that were utilized in the investigation performed with each coin.

\subsection{Stress-strain curve}

The stress-strain curve of the Copper-Nickel alloy (Cu75Ni25) was determined by means of the stack compression test [22]. This test was chosen instead of the conventional tensile test due to its capability to characterize material stress response, without necking, directly from the coin blanks up to values of 0.6 that are commonly found in coin minting.

The stack compression test was performed on multi-layer cylinder specimens that were assembled by piling up four circular disks with $15 \mathrm{~mm}$ diameter machined out of the coin blanks utilized in production. The tests were carried out at room temperature on a hydraulic testing machine (Instron SATEC $1200 \mathrm{kN}$ ) with a cross-head speed equal to $10 \mathrm{~mm} /$ min. The photograph included in Figure 5 shows a multilayer cylinder test specimen before and after compression.

The experimentally determined stress-strain curve of Cu75Ni25 and its approximation by means of the LudwikHollomon's strain hardening model are shown in Figure 5,

$$
\sigma=741.2 \varepsilon^{0.41}(\mathrm{MPa})
$$



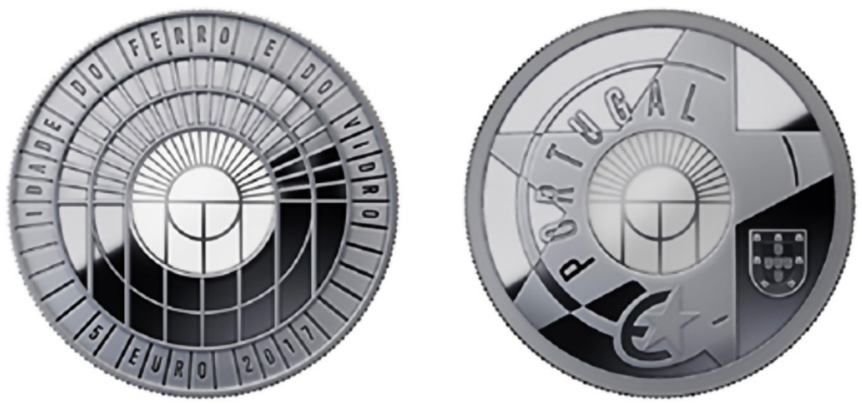

(a)
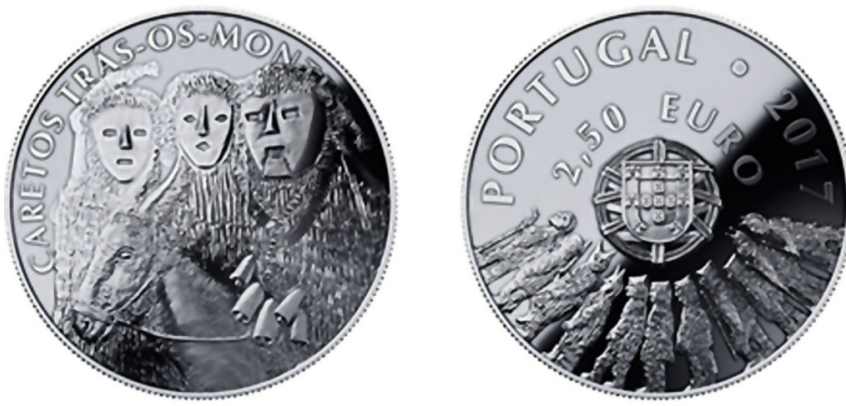

(b)

Fig. 4. Test cases utilized in numerical simulation and experimentation with Cu75Ni25 coin blanks. (a) Collection coin dedicated to the age of iron and glass in Europe ("coin A"), (b) Collection dedicated to Portuguese Ethnography ("coin B").

\subsection{Validation of the industrial force measurements}

The coin minting experiments were performed in a Schuler (MRV300) knuckle-joint press available at the Portuguese Mint.

The force measurements read in the control panel of the knuckle-joint press were validated against those obtained in the hydraulic testing machine equipped with a calibrated load-cell. The validation was accomplished by compressing five disks made from $\mathrm{Ag} 500$ ( $\mathrm{Ag} 50 \mathrm{Cu} 50$ ) between flat parallel dies in the knuckle-joint press for different amounts of stroke and by compressing two other disks of the same alloy between the same flat parallel dies up to the maximum amount of stroke in the hydraulic testing machine. Table 2 provides information on the experimental work plan utilised for validating the forces provided by the knuckle-joint press and the enclosed photograph shows the dies and a disk before and after compression.

The disks utilized in these verification tests had $27.6 \pm 0.05 \mathrm{~mm}$ diameter and $1.65 \mathrm{~mm}$ thickness and were machined out of the Ag500 coin blanks utilized in production. The surfaces of the flat dies had an average roughness $\mathrm{Ra}=0.319 \mu \mathrm{m}$ in order to allow measuring compression forces up to $800 \mathrm{kN}$, because in case surfaces were polished with a quality similar to that utilized in coin minting dies the values of force would be much smaller, as it will be shown.

The black solid circular markers in Figure 6 are the maximum forces read in the control panel of the industrial knuckle-joint press for the five compression disks labelled as " $1-5$ " in Table 2. The grey open diamond markers correspond to the average force vs. stroke evolution for the two disks labelled as "6" and "7" in Table 2 obtained from continuous upset compression in the hydraulic testing machine. As shown, the overall agreement is good and enables the utilization of the forces measured by the industrial knuckle-joint press with minor corrections.

Finite element analysis of the upset compression of the disks also allow concluding that the rough surface of the flat dies constrains material outward flow with friction shear stresses corresponding to a friction factor $m \cong 0.35$ (refer to the black dashed line in Fig. 6). This type of surface finishing was needed to ensure that upset compression forces were in the range of coin minting forces because flat parallel dies with typical surface finishing of that utilized in coin minting dies would only be able to provide forces up to $300 \mathrm{kN}$ (refer to the black open rectangular markers and to the disk labelled as "8" in Table 2).

\section{Results and discussion}

This section starts with the assessment of the accuracy and reliability of the finite element predictions against measurements and observations from coin minting experiments with coin "A" and ends by disclosing a finite element design based procedure for correcting the die profiles. This last presentation made use of coin "B".

\subsection{Filling of the relief coin features}

The numerical simulation of the coin minting process allows analysing the filling of the relief coin features before fabricating the dies and producing the coins. One of the variables that can be used for this purpose is the finite element predicted distribution of z-stress $\sigma_{z}=-p$ (where, $p$ is the applied contact pressure) in the obverse and reverse of the coins at different percentages of the total die stroke.

This is shown in Figure 7 for coin "A". The dark red colour in the distribution of z-stress corresponds to pressures close to zero and, therefore, to regions of the coins that are not in contact with the dies. In contrast, the other colours evolving from light red to dark blue correspond to increasing values of the applied pressure and, therefore, to regions of the coins that are in contact with the dies. In addition to this, the enclosed photographs allow distinguishing the actual regions of the obverse and reverse of the coins that are in contact with the dies (refer to the shiny surfaces) from those that are still free. In each experimental test, the coins were intentionally struck one single time in order to prevent possible defects resulting from multiple strikes. At least two repetitions were made for each testing condition. The agreement of the photographs with the finite element predicted distribution of zstress for the same amount of die stroke is very good.

Other conclusions that may be drawn from the analysis of the z-stress are; (i) the identification of the regions of the coin where contact starts and (ii) the detection of excessive lack of symmetry during coin minting. Ideally, contact should initiate at the centre of the coin and progressively evolve towards its rimmed edge and should develop as much symmetric as possible in order to minimize bending moments. 
Table 1. Material and geometry of the coin blanks utilized in the test cases.

\begin{tabular}{lllll}
\hline & Material & $\begin{array}{l}\text { Diameter } \\
(\mathrm{mm})\end{array}$ & $\begin{array}{l}\text { Thickness } \\
(\mathrm{mm})\end{array}$ & $\begin{array}{l}\text { Edge } \\
\text { Thickness }(\mathrm{mm})\end{array}$ \\
\hline $\begin{array}{l}\text { Age of iron and glass } \\
\text { ("coin A") }\end{array}$ & Copper-Nickel alloy (Cu75Ni25) & $29.65 \pm 0.05$ & 2.24 & $2.79 \pm 0.1$ \\
$\begin{array}{l}\text { Portuguese Ethnography } \\
\text { ("coin B") }\end{array}$ & Copper-Nickel alloy (Cu75Ni25) & $27.65 \pm 0.05$ & 1.84 & $2.28 \pm 0.1$ \\
\hline
\end{tabular}

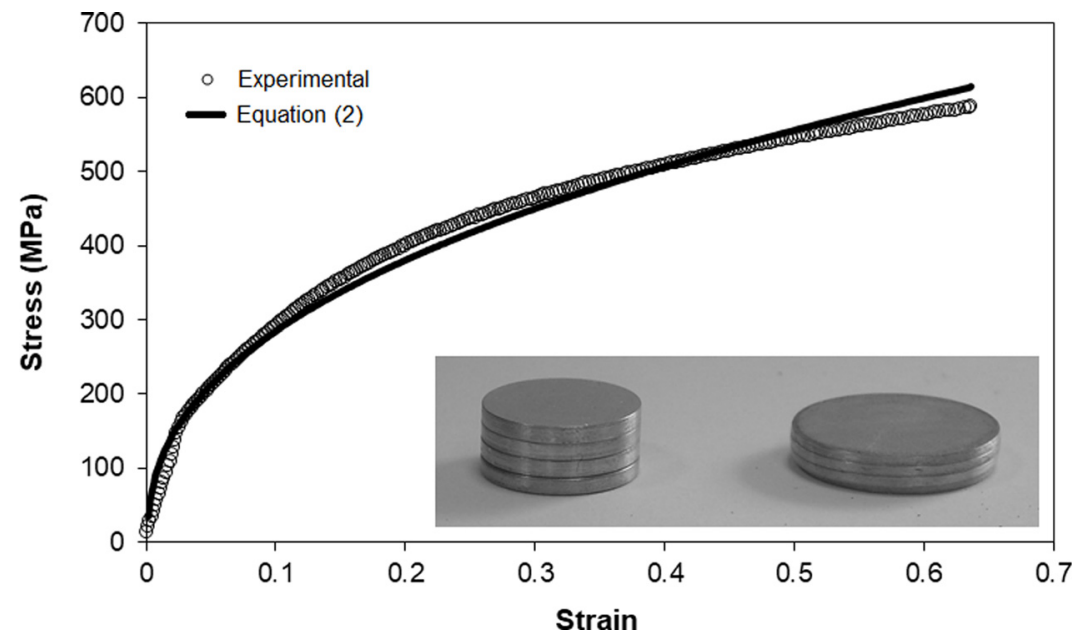

Fig. 5. Stress-strain curve of Copper-Nickel (Cu75Ni25) coin blanks. The solid line is the Ludwik-Hollomon's approximation given by equation (2).

Table 2. Experimental procedure for validating the forces measured in the knuckle-joint press with a photograph of the disks before and after compression.

\begin{tabular}{|c|c|c|c|c|c|}
\hline Disk & Equipment & Die roughness $(\mu \mathrm{m})$ & Diameter $(\mathrm{mm})$ & Thickness $(\mathrm{mm})$ & Compression stroke $(\mathrm{mm})$ \\
\hline 1 & \multirow{6}{*}{ Knuckle-joint press } & \multirow{7}{*}{0.319} & 27.61 & 1.639 & 0.100 \\
\hline 2 & & & 27.60 & 1.643 & 0.144 \\
\hline 3 & & & 27.62 & 1.653 & 0.227 \\
\hline 4 & & & 27.63 & 1.642 & 0.331 \\
\hline 5 & & & 27.63 & 1.644 & 0.370 \\
\hline 6 & & & 27.61 & 1.646 & 0.380 \\
\hline 7 & \multirow[t]{2}{*}{ Hydraulic testing machine } & & 27.63 & 1.656 & 0.380 \\
\hline 8 & & $<0.01$ & 27.63 & 1.646 & 0.380 \\
\hline
\end{tabular}




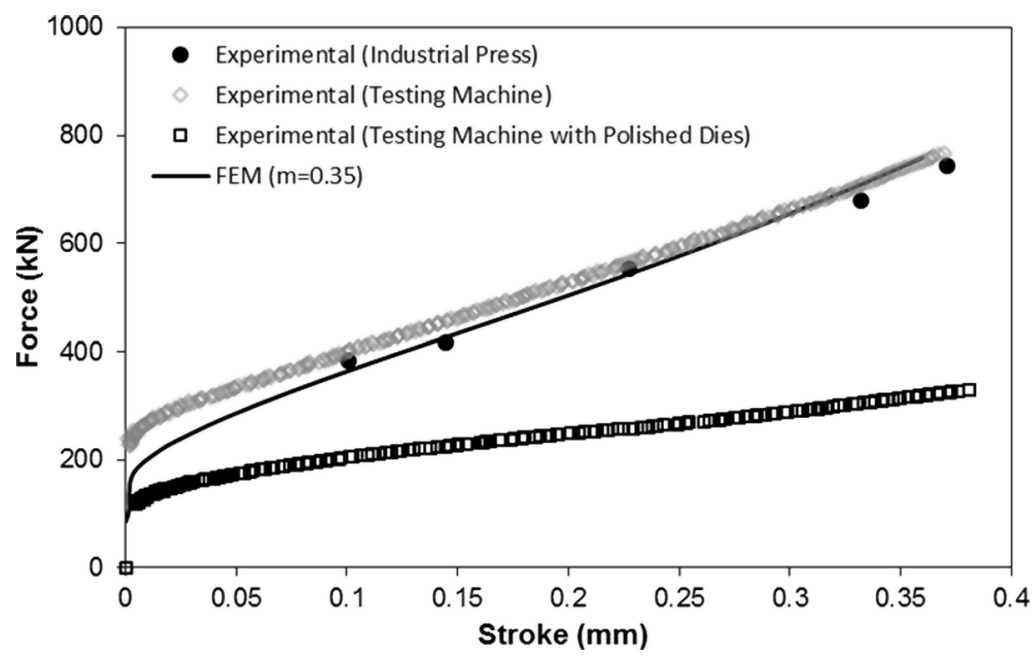

Fig. 6. Force vs. stroke results for the upset compression of disks between flat parallel dies performed in the industrial knuckle-joint press and in the hydraulic testing machine. Finite element (FEM) results are included for reference purposes.

The results shown in Figure 7 allow concluding that contact initiates at the rimmed edge of the disk due to the flat edge profile of the reverse die and to the large thickness of the disk edge. However, the distribution of pressure is symmetric meaning that the resultant vertical force is aligned with the vertical symmetry line of the die and that the bending moments applied in the dies at the end of stroke should be small. The conclusion is that the die profiles of the "coin A" do not need to be corrected.

\subsection{Force vs. die stroke evolution}

Figure 8 presents the finite element predicted evolution of the force vs. die stroke and the actual forces measured in the industrial knuckle-joint press for coin "A". The forces in the industrial knuckle-joint press were measured for different values of die stroke and are plotted as open circular markers.

The force vs. die stroke evolution pattern is typical of close-die forging, but coin minting requires high compression forces with relatively small die strokes. The force is low until every surface of the disk are squeezed and material is trapped to start filling the remaining and more intricate details in the dies (refer to 'P1' in Fig. 8). As the dies continue to close, the force increases sharply to "P2" at which the relief coin features are completely filled with sharp corners, well-defined features and a brilliant surface finish.

The last positions "P3" above the horizontal dashed line were obtained for die strokes beyond needed and gave rise to localized plastic deformation of the tooling and to the production of coins with final diameters slightly larger than that originally specified $(30 \mathrm{~mm})$.

\subsection{Correction of the die profile}

Coin minting with lack of contact symmetry can be optimized by correcting the die profile. In practical terms the optimization consists of tilting the obverse and reverse die reliefs so that the centre of die pressure is located on the vertical symmetry line of the die.

The procedure for correcting the die profile is performed for a die stroke corresponding to the end of coin minting and involves the following tasks to be carried out for each die:

- Calculate the point of application $\left(x_{r}, y_{r}\right)$ of the resultant vertical force $F_{r}$ for the original (non-corrected) die profile;

- Perform a first correction of the original die profile by tilting the obverse and reverse die reliefs in the $\mathrm{x}$ and $\mathrm{y}$ directions (Fig. 9) and determine the corresponding new point of application $\left(x_{r}^{1}, y_{r}^{1}\right)$ of the resultant vertical force $F_{r}^{1}$

- Repeat b) using additional correction values until the resultant vertical force $F_{r}^{n}$ after correction $n$ is located at the centre (symmetry line) $\left(x_{r}^{n}, y_{r}^{n}\right) \cong(0,0)$.

The point of application $\left(x_{r}^{i}, y_{r}^{i}\right)$ of the resultant vertical force $F_{r}^{i}$ after correction $i$ is determined from,

$$
x_{r}^{i}=\frac{\sum_{j=1}^{N} F_{j}^{i} x_{j}^{i}}{\sum_{j=1}^{N} F_{j}^{i}} \quad y_{r}^{i}=\frac{\sum_{j=1}^{N} F_{j}^{i} y_{j}^{i}}{\sum_{j=1}^{N} F_{j}^{i}} .
$$

where, $F_{j}^{i}$ is the vertical force of a nodal point $j$ with coordinates $\left(x_{j}^{i}, y_{j}^{i}, z_{j}^{i}\right)$ located on the surface of the disk in contact with the die after correction $i$ of the original die profile, and $N$ is the total number of nodal points on the surface of the disk in contact with the die. The resultant vertical force $F_{r}^{i}$ after correction $i$ is determined from,

$$
F_{r}^{i}=\sum_{j=1}^{N} F_{j}^{i}
$$

The above mentioned procedure was applied to "coin B" and the resultant vertical force $F_{r} \cong 1200 \mathrm{kN}$ for the 

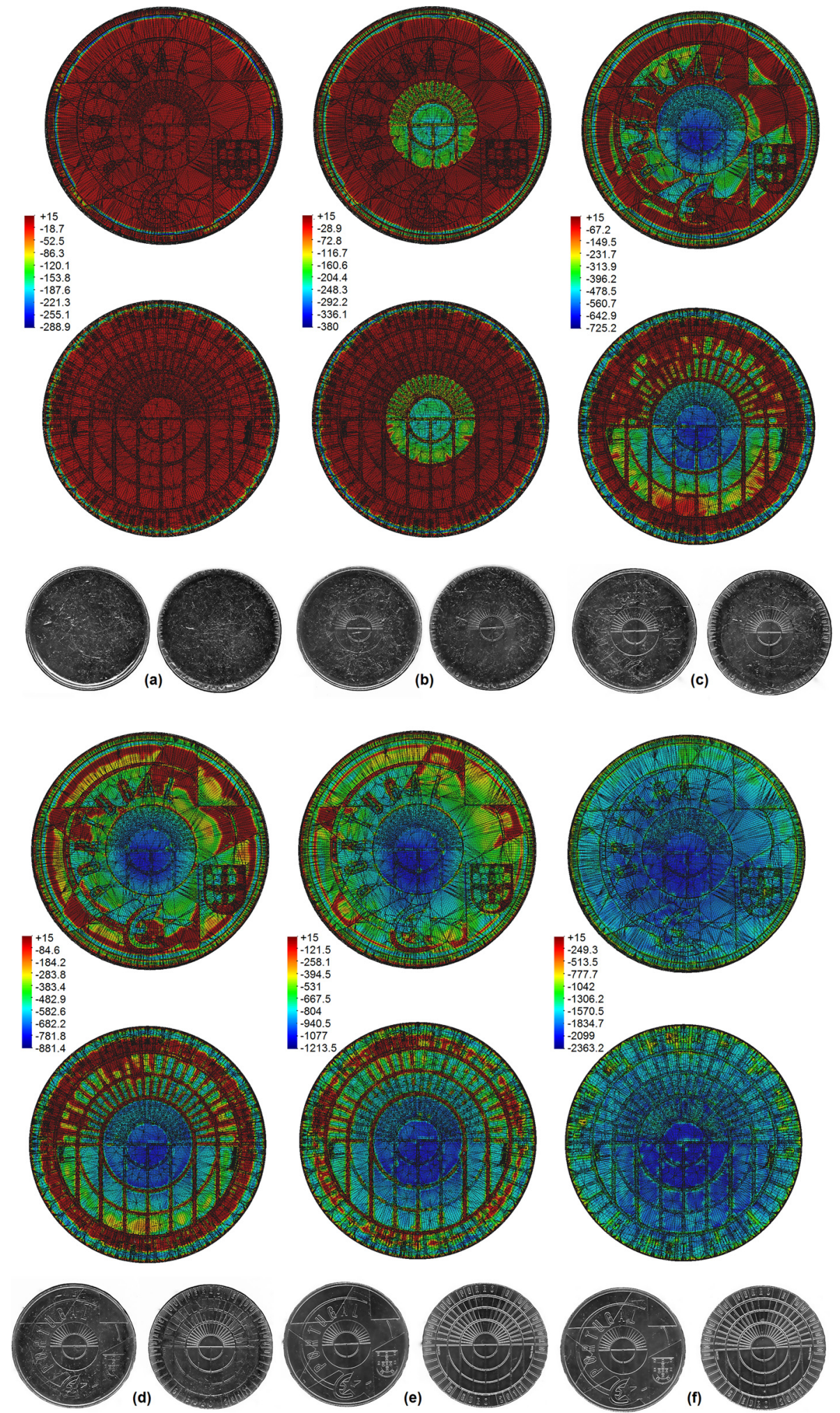

Fig. 7. Finite element predicted distribution of z-stress (MPa) at (a) $17 \%$, (b) $35 \%$, (c) $76 \%$, (d) $85 \%$, (e) $94 \%$ and (f) $100 \%$ of the total die stroke with photographs of the corresponding samples ("coin A").

original (non-corrected) die profile obtained from the clay models supplied by the sculptor was obtained from the finite element estimate of force at the end of die stroke (refer to point 'P' in Fig. 10).
The point of application $\left(x_{r}, y_{r}\right)$ of the resultant vertical force $F_{r}$ is plotted in Figure 11 and the misalignment towards the vertical symmetry line of the die is approximately equal to $0.16 \mathrm{~mm}$ and $0.83 \mathrm{~mm}$ in the $\mathrm{x}$ 


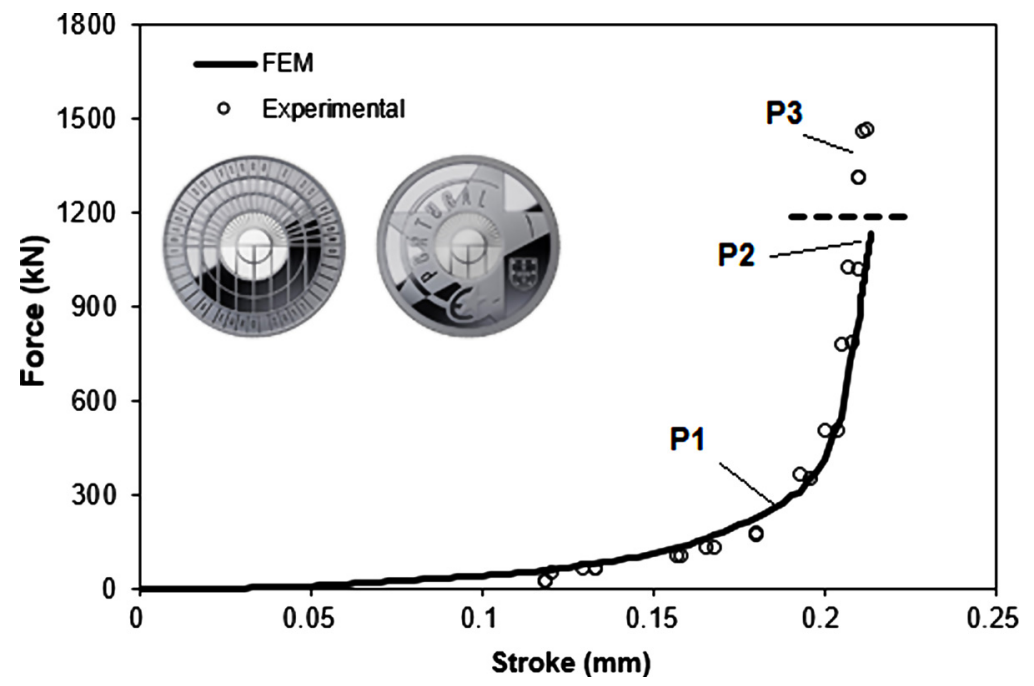

Fig. 8. Experimental and finite element predicted evolution of the force with die stroke for "coin A".

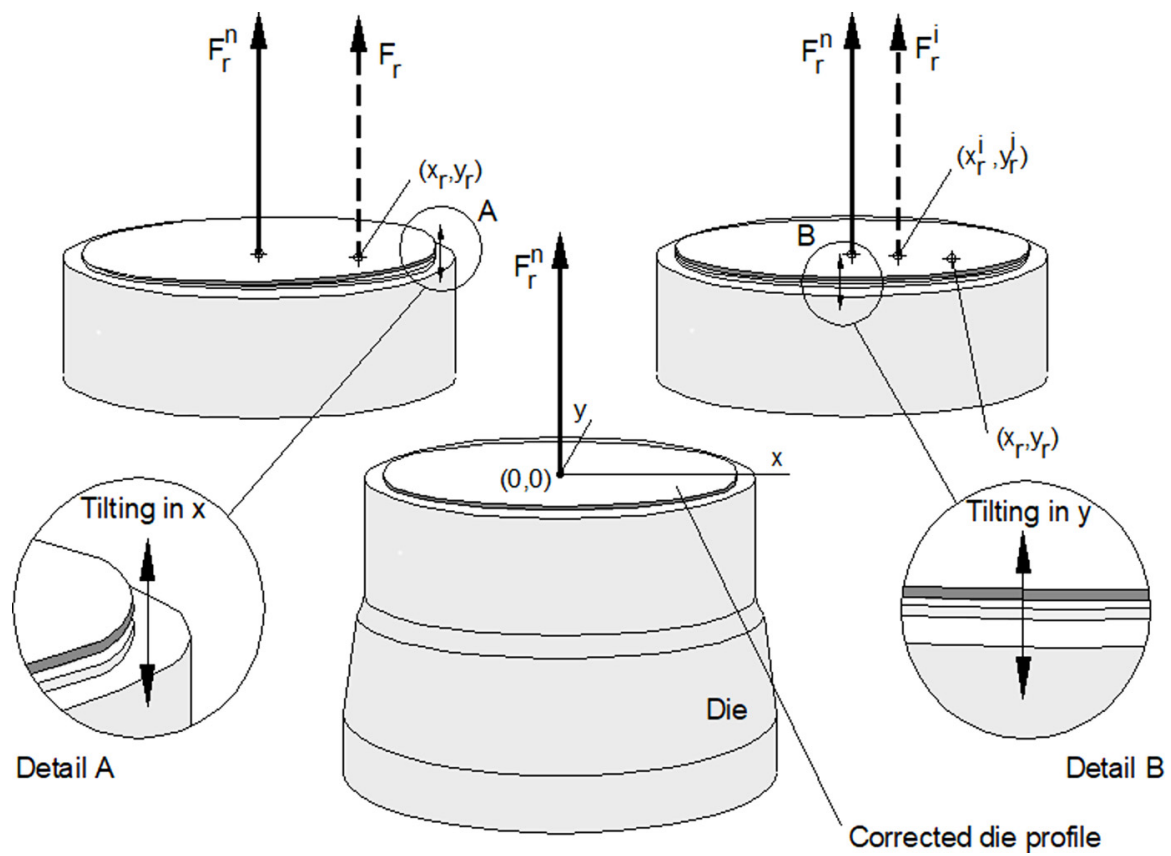

Fig. 9. Schematic representation of the tilting of the die reliefs in the $\mathrm{x}$ and y directions for ensuring that the resultant vertical force $F_{r}^{n}$ after correction $n$ is located at the symmetry line $(0,0)$.

and y directions. This gives rise to the following bending moment $M$ applied in both dies,

$$
M=M_{x} \vec{e}_{x}+M_{y} \vec{e}_{y}=996 \vec{e}_{x}+192 \vec{e}_{y}(\mathrm{Nm})
$$

After three iterations with the proposed die correction procedure the obverse and reverse die reliefs are tilted by $10 \mu \mathrm{m}$ and $60 \mu \mathrm{m}$ in the $\mathrm{x}$ and $\mathrm{y}$ directions and the resulting bending moment of equation (5) is reduced to a value 9 times smaller,

$$
M=M_{x} \vec{e}_{x}+M_{y} \vec{e}_{y}=132 \vec{e}_{x}+24 \vec{e}_{y}(\mathrm{Nm})
$$

The overall misalignment of the resultant vertical force towards the vertical symmetry line of the die is also reduced to $0.02 \mathrm{~mm}$ and $0.11 \mathrm{~mm}$ in the $x$ and $y$ directions (refer again to Fig. 10),

Figure 12 presents a comparison of the finite element predicted distribution of z-stress for the original and final corrected die profiles for the die stroke at the end of coin minting. As seen, the corrected die profiles improve the overall symmetry of the coin minting process.

Experiments performed at the Portuguese Mint confirmed that the coining force is $100 \mathrm{kN}$ smaller in case of the corrected die profiles. This is due to a reduction of the 


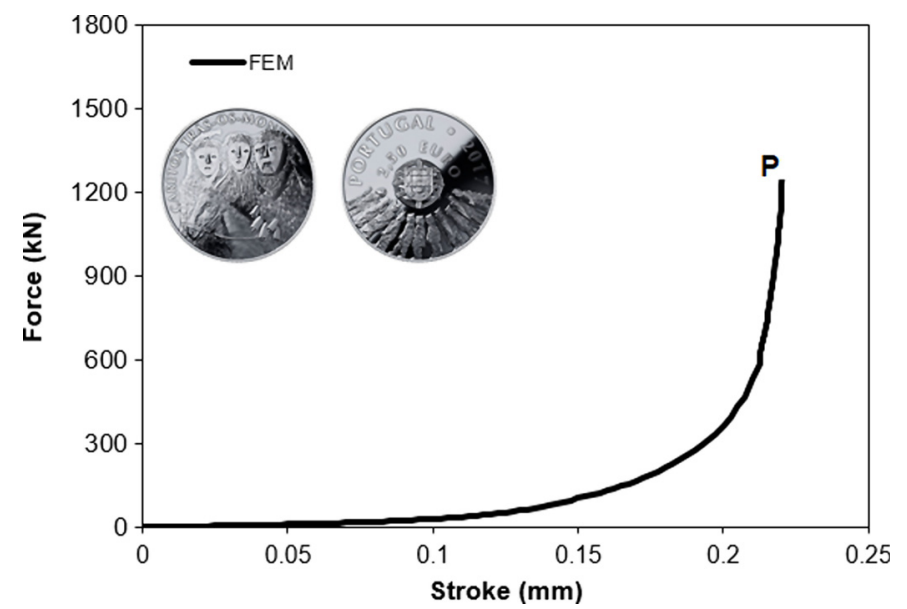

Fig. 10. Finite element predicted evolution of the force with die stroke for "coin B".
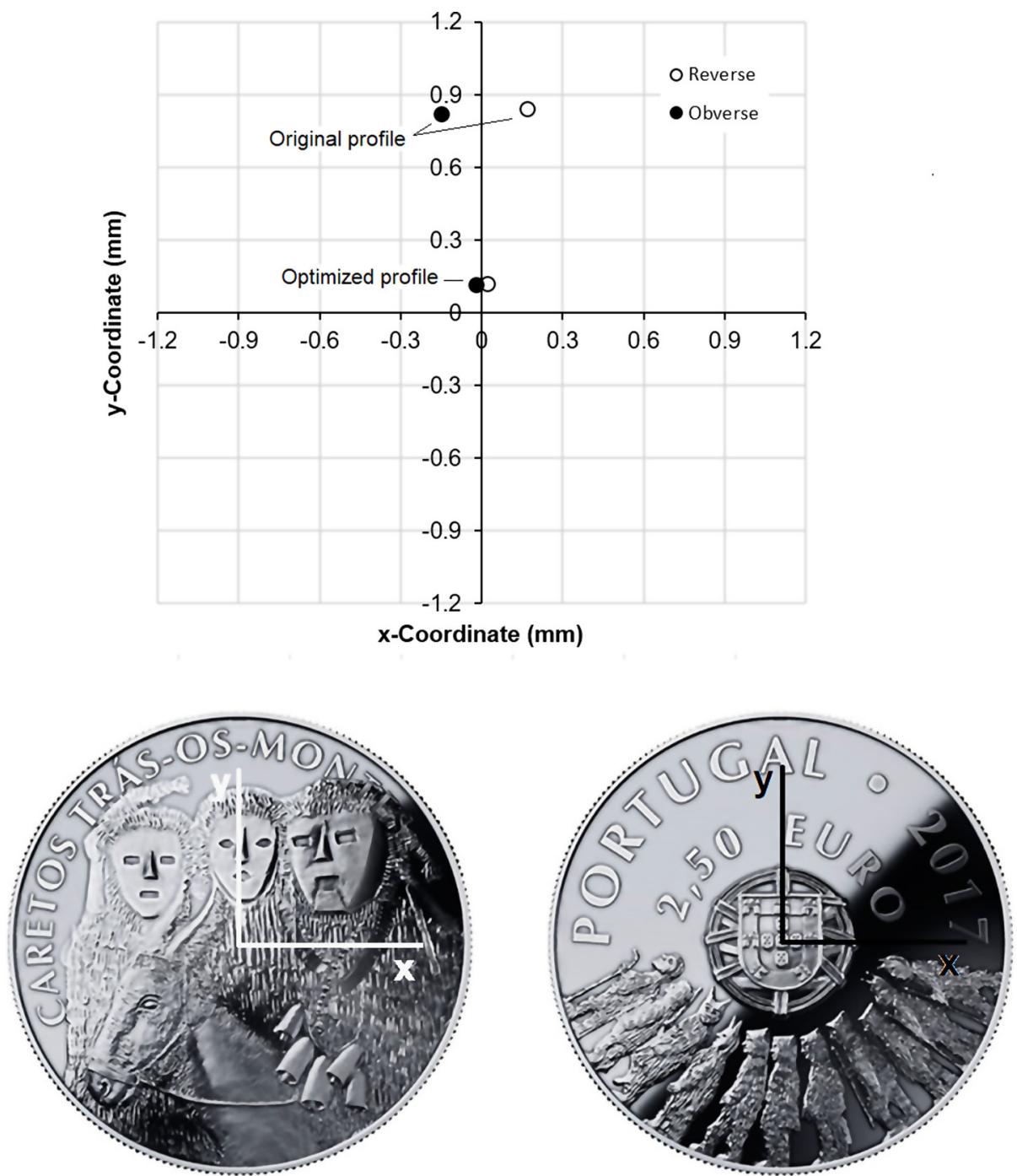

Fig. 11. $x, y$ position of the resultant vertical force in the reverse and obverse dies for the original and final corrected die profiles ("coin B"). 

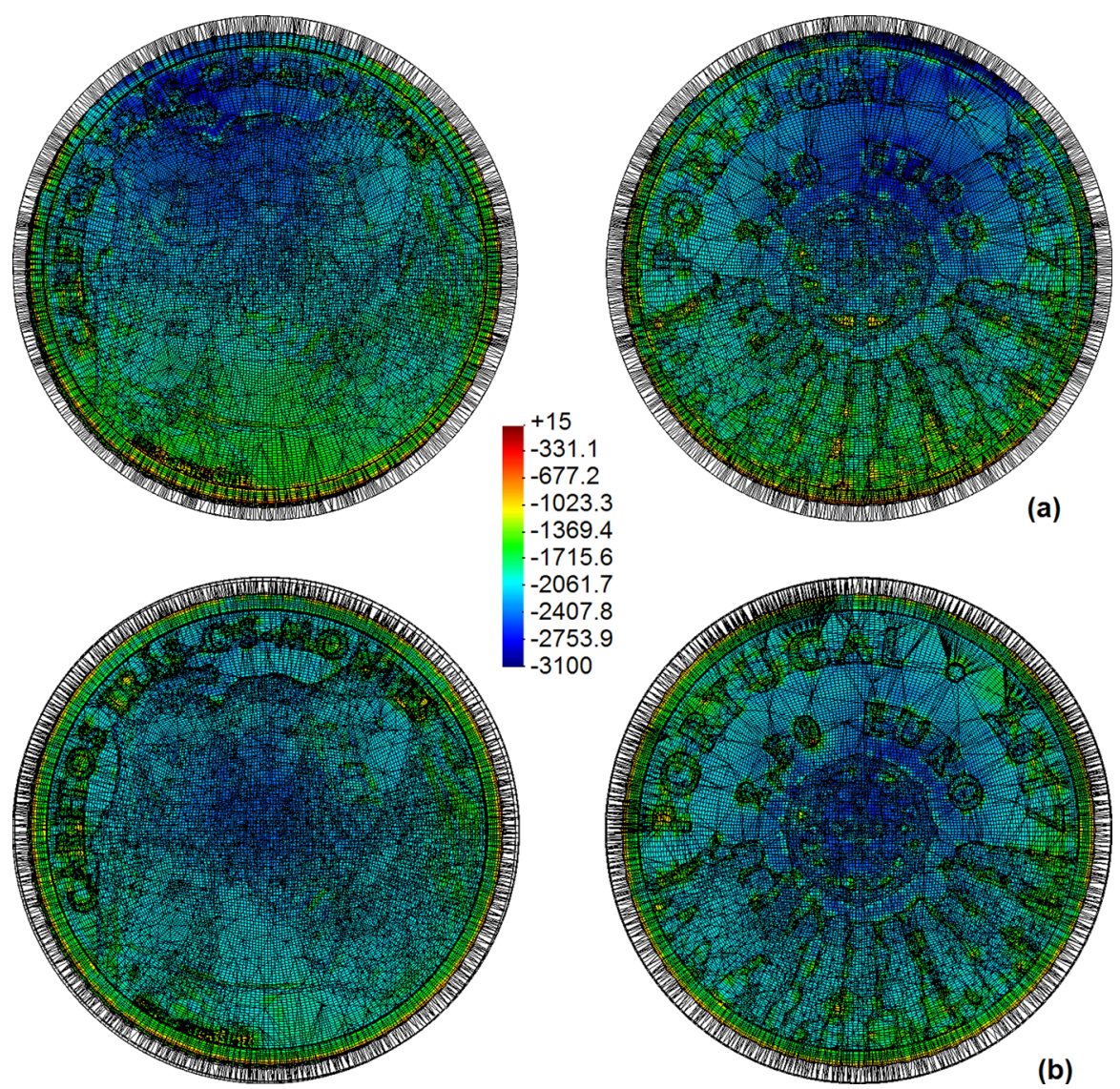

Fig. 12. Finite element predicted distribution of z-stress (MPa) at the end of coin minting for the (a) original die and for (b) corrected die profiles ("coin B").

maximum z-stress from $-3100 \mathrm{MPa}$ (in the original die profiles, Fig. 12a) to $-2840 \mathrm{MPa}$ (in the corrected die profiles, Fig. 12b).

Preliminary experiments with several other collection coins confirmed an increase in die life of approximately 2 times by using the new finite element design based procedure for correcting the die profiles.

\section{Conclusions and future perspectives}

The accuracy and reliability of a finite element computer program being developed by the authors in collaboration with the Portuguese Mint was successfully assessed by comparing numerical and experimental results of the coin minting forces and of the filling of the relief coin features.

A new experimental procedure based on the stack compression test was successfully utilized to determine the stress-strain curve directly from the coin blanks. The specimens utilized in the test consisted of multilayer cylinders that were assembled by piling up circular disks machined out of the coin blanks utilized in production.

The innovative finite element based design procedure for correcting the die profiles shows that numerical simulation can be utilized to optimize shapes, reduce coin minting forces and extend die life. The procedure can also be utilized to foster the relationship between artists who create the modulated reliefs of the coins and the technicians and engineers who fabricate the dies and industrialize the process with the objective of minimizing defects and reducing try-outs.

Luis Alves and Paulo Martins would like to acknowledge the support provided by the Portuguese Mint (Imprensa Nacional Casa da Moeda) and IDMEC under LAETA-UID/EMS/50022/ 2013.

The authors would also like to acknowledge the technical assistance of Elisabete Novais and Nuno Caetano from Imprensa Nacional Casa da Moeda. The support of Dr. Silvia Garcia and Dr. Alcides Gama from Imprensa Nacional Casa da Moeda is also acknowledged.

\section{References}

1. Y. Bocharov, S. Kobayashi, E.G. Thomsen, J. Eng Ind Trans ASME 84 (1962) 491-501

2. N. Bay, T. Wanheim, Wear 38 (1976) 225-234

3. C. Kiran, M.C. Shaw, Ann. CIRP 32 (1983) 151-154

4. F. Delamare, P. Montmitonnet, J. Mech. Work Technol 10 (1984) 253-271

5. W.A.M. Brekelmans, L.H.G. Mulders, J.A.H. Ramaekers, Ann. CIRP 37 (1988) 235-238

6. M.J.M. Barata Marques, P.A.F. Martins, J. Mater. Process. Technol. 26 (1991) 337-348. 
7. P.J. Leitão, A.C. Teixeira, J.M.C. Rodrigues, P.A.F. Martins, J. Mater. Process. Technol. 70 (1997) 178-184

8. A.C. Teixeira, P.J. Leitão, P.A.F. Martins, A multimetallic foil technology for minting medals and coins with two or three colors, EP - 97901854, European Patent Office, 1999

9. H.H. Choi, J.H. Lee, S.K. Bijun, B.S. Kang, J. Mater. Process. Technol. 72 (1997) 396-402.

10. H. Ike, M. Plancak, J. Mater. Process. Technol. 80-81 (1998) 101-107

11. G. Buffa, L. Fratini, F. Micari, in Proceedings of NUMIFORM 2007 - Materials Processing and Design: Modelling, Simulation and Applications (AIP Conference Proceedings 2007) 908 1005-1010

12. J.P. Xu, Y.Q. Liu, S.Q. Li, S.C. Wu, Comput. Model. Eng. Sci. 38 (2008) 201-215.

13. K. Guo, PhD thesis, Carleton University, Ontario, Canada, 2009

14. W. Zhong, Y. Liu, Y. Hu, S. Li, M. Lai, Int. J. Adv. Manuf. Technol. 63 (2012) 939-953.
15. Q. Li, W. Zhong, Y. Liu, Z. Zhang, Int. J. Mech. Sci., 128-129 (2017) 105-115.

16. K. Shirasaka, in Proceedings of the 29th. Mint Directors Conference, Bangkok, Thailand, 2016

17. P. Alexandrino, P.J. Leitão, L.M. Alves, P.A.F. Martins, J Mater: Des Appl (2017), accepted for publication, DOI: $10.1177 / 1464420717709833$

18. M.J.M. Barata Marques, P.A.F. Martins, Int. J. Numer. Methods Eng. 30 (1990) 1341-1354.

19. C.V. Nielsen, W. Zhang, L.M. Alves, N. Bay, P.A.F. Martins, in Modelling of thermo-electro-mechanical manufacturing processes with applications in metal forming and resistance welding, Springer-Verlag, London, UK, 2013

20. A.O. Cifuentes, A. Kalbag, Finite Elem. Anal. Des. 12 (1992) 313-318.

21. S.E. Benzley, E. Perry, K. Merkley, B. Clark, G. Sjaardema, in Proceedings of the Fourth International Meshing Roundtable, 179-191, 1995.

22. L.M. Alves, C.V. Nielsen, P.A.F. Martins, Exp. Mech. 51 (2011) 1565-1572.

Cite this article as: Paulo Alexandrino, Paulo J Leitão, Luis M Alves, Paulo A.F. Martins, Finite element design procedure for correcting the coining die profiles, Manufacturing Rev. 5, 3 (2018) 